\title{
An Assembling Procedure for DNA Bipyramids with Minimum Component Strands
}

\author{
Tao Deng ${ }^{1,2,3, *}$, Mengge Huang ${ }^{1}$, Jingyi Zhang ${ }^{1}$ \\ ${ }^{1}$ Key Laboratory of China's Ethnic Languages and Information Technology of Ministry of \\ Education, Northwest Minzu University, Lanzhou 730030, P. R. China. \\ ${ }^{2}$ Key Laboratory of Streaming Data Computing Technologies and Application, Northwest \\ Minzu University, Lanzhou 730030, P. R. China. \\ ${ }^{3}$ School of Mathematics and Computer Science, Northwest Minzu University, Lanzhou \\ 730030, P. R. China \\ *Corresponding author: dttom@1zu.edu.cn
}

(Received November 10, 2020)

\begin{abstract}
DNA cages are ideally suited to make nanostructures which serve as containers for drug delivery. Using fewer strands to assemble DNA cages is of importance to the design of DNA molecules with multiple component strands. In this study, we propose a rational assembling procedure to design and analyze DNA bipyramids with minimum strands. The results show that the odd-half twist has a major impact on assembling strands required to construct DNA cages and this method could offer a search of DNA bipyramids with minimum component strands faster. This research provides new insights into design and synthesis for DNA bipyramid-like cages from mathematical perspective.
\end{abstract}

\section{Introduction}

DNA has long been regarded not only as hereditary materials but also as raw materials for applications in nanostructures ${ }^{[1,2]}$. Over the past decades, DNA molecules with desired shapes have already been designed and synthesized experimentally ${ }^{[3]}$, such as tetrahedra [4-6], octahedron ${ }^{[7,8]}$, truncated octahedra ${ }^{[9,10]}$, bipyramid ${ }^{[11]}$, prims ${ }^{[12,13]}$. These wireframed, cagelike nanostructures, assembled by multiple DNA single strands, are now considered 
increasingly important materials for biosensors and drug delivery ${ }^{[14-18]}$. Meanwhile, it is worth noting that some designed DNA molecules with required topological structures play crucial roles in overcoming their challenges of structural stability and yield. Also, low cost and simple structures require good assembling methods in synthesizing DNA nanostructures. One practical solution to reduce the complexity of theses entangled structures is to use component strands as few as possible ${ }^{[19-22]}$. Therefore, it is necessary to clarify the procedure to reduce the number of strands assembled in DNA molecules. Bipyramids, more complex than Platonic solids, have been synthesized early in 2007 by using six DNA single strands ${ }^{[11]}$ and have been used as a new class of SPECT molecular probes ${ }^{[23]}$. Our study is aiming at an assembling procedure for DNA bipyramids with fewest component strands from a theoretical viewpoint.

To better understand the problem, we need to resort to some basic knowledge of mathematics. From a topological perspective, DNA strands could be interpreted as thin lines without thickness and each DNA polyhedron is a polyhedral link which turns the DNA single strand into the component of the link ${ }^{[24,25]}$. Thus, DNA polyhedral link serves as an ideal mathematical model to characterize DNA polyhedra structure ${ }^{[26]}$. Then, a series of studies based on this model successfully were carried out to explain some intrinsic properties regarding the assembly mechanism of DNA polyhedra ${ }^{[27-32]}$. Some of these polyhedral links have been used as templates to construct $3 \mathrm{D}$ molecular objects as well ${ }^{[31,33,34]}$. However, this model pays more attention to the relationship between mathematical variables and becomes weak in actual use. Using fewer DNA strands to assemble polyhedra is one of the most important practical issues, for it could result in the low cost and good stability of production. Recent studies have showed that the odd half-twist has a major impact on the reduction the number of required strands ${ }^{[35,36]}$. Furthermore, the positional distribution of odd-half turn DNA strands is discussed to predict and design DNA configurations of less DNA strands at the structural level ${ }^{[21]}$. Oddhalf turn makes it possible for strands to enter neighbouring face, reducing the number of component strands to construct DNA. Thus, it is of importance to clarify how to introduce oddhalf turn and where to place it. This procedure enables us to generate DNA polyhedral links easier and faster.

In the previous study, we have constructed and analysed DNA polyhedra from through computation program ${ }^{[37,38]}$. Here, we present an assembling procedure for DNA bipyramids with minimal component strands based on computer program. This procedure would search for all permissible topological structures satisfying DNA constructing rules, and select the ones with minimum number of strands. 


\section{Methods}

\subsection{Vertex and edge}

From the viewpoint of mathematics, the cage-like DNA architectures actually consist of interlocked and interlinked DNA strands. In these structures, three-, four-, and five-arm junctions, shown in Figure 1, occur on the vertices of polyhedra, corresponding to degree-three, degree-four, and degree-five vertex, respectively. Note that the two strands of DNA are antiparallel.

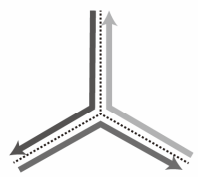

(a)

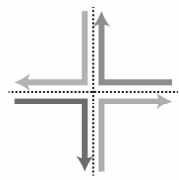

(b)

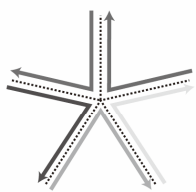

(c)

Figure 1. Three basic vertex junctions: (a) degree-three vertex; (b) degree-four vertex; (c) degree-five vertex.

Besides, twisted DNA strands, which form the edges of polyhedra, include half-twists of two types: $2 n+1$ half-twists and $2 n$ half-twists. $2 n+1$ half-twists (Figure 2a left) can be simplified as odd half-twist (Figure 2a middle), while $2 n$ half-twists (Figure $2 \mathrm{~b}$ left) can be simplified as even half-twist (Figure $2 b$ middle). We can find that the odd half-twist changes the direction of strands (Figure 1a right), but the even half-twist doesn't ${ }^{[24,39]}$ (Figure 2b right). If each edge of a polyhedron is twisted with even half-turns, the number of strands equals its face number ${ }^{[27]}$. On the other hand, with the odd half-twists available, the strand number is uncertain and less than the face number ${ }^{[32]}$.

(a)
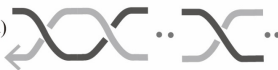

$2 n+1$ half-twists

(b)

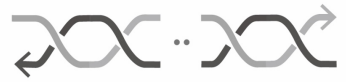

$2 n$ half-twists
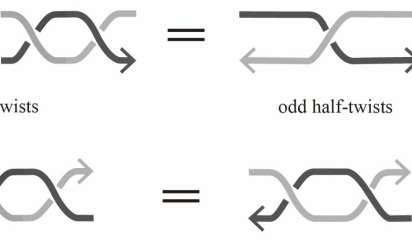

even half-twists

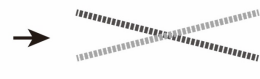

direction of strands changes

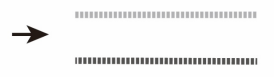

direction of strands remains

Figure 2. Two types for twisted DNA strands: (a) $2 n+1$ half-twists corresponds to the odd halftwists (turn) and its direction of strands changes; (b) $2 n$ half-twists corresponds to the even half-twists and its direction of strands remains. 
A tetrahedron example is given to specify the two cases of the sole even half-twists and the mix of even and odd half-twists. Figure 3 a shows a tetrahedron with even half-twists, where its faces and strands are equal in number; Figure $3 \mathrm{~b}$ shows a tetrahedron with mix of the odd half-twists and the even half-twists, where the strands are less than faces in number. Intuitively, the emergence of the odd half-twists has brought the reduction of strands within the bounds of possibility.

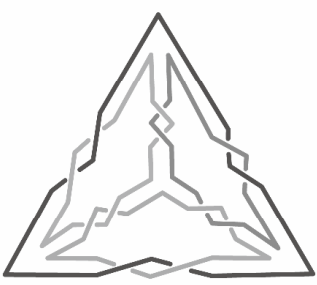

(a)

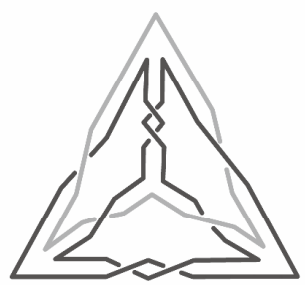

(b)

Figure 3. Two ways to construct a DNA tetrahedron: (a) a tetrahedron with sole even half-turns (4 strands); (b) a tetrahedron with mix of the odd half-twist and the even half-twist (2 strands).

\subsection{Constructing rule}

R. Twarock et al. utilized bead configurations to construct DNA dodecahedral and icosahedral cages with a minimal number of strands ${ }^{[35,36]}$. A DNA dodecahedron or icosahedron requires at least two circular strands and each face of the polyhedron must have even number of crossings. Under this constructing rule, each face of the bipyramid must have an even number of half-twists. This ensure that the two oriented strands of each edge are antiparallel or oppositely oriented, satisfying structures of the DNA double helix.

\subsection{Generate-and-test paradigm}

In this step, we must search for all possible types for a DNA bipyramid. In other words, we need to generate all possible links of a bipyramid, and then test them for DNA strands according to DNA constructing rule. This step is referred to as generate-and-test paradigm, further performed in 2.5 Program. 


\subsection{Label the diagram}

Bipyramid mainly includes triangular bipyramid and pentangular bipyramid, and Figure 4 shows both with labels, in which capital letter stands for its face, lowercase letter for its edge. Specifically, the lowercase letters are used to tell in detail what type of twisted strands to be placed on the edges.

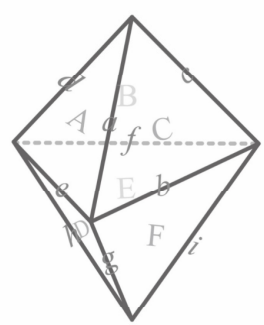

(a)

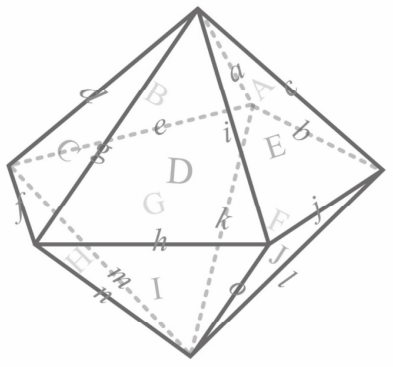

(b)

Figure 4. Diagrams with labels: (a) triangular bipyramid; (b) pentangular bipyramid.

\subsection{Program}

The code in Python for triangular bipyramid is as follows, and it is also applied to the pentangular bipyramid with only minor modifications.

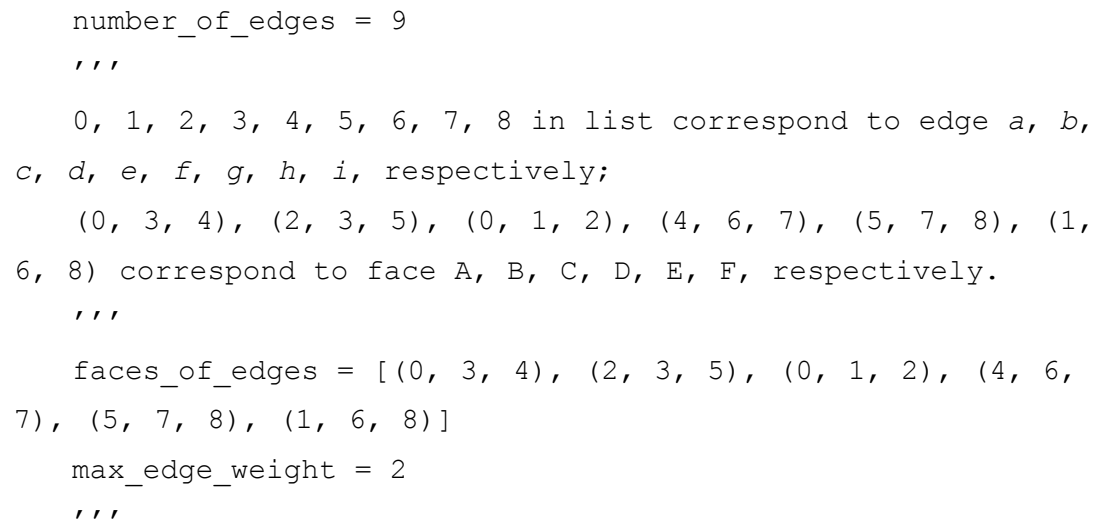


Generate a binary table of 1 and 2, traversing the cases of even and odd for a polyhedron. The function of increase adds 1 to the binary table on each call, ensuring that all combinations of 1 and 2 can be traversed.

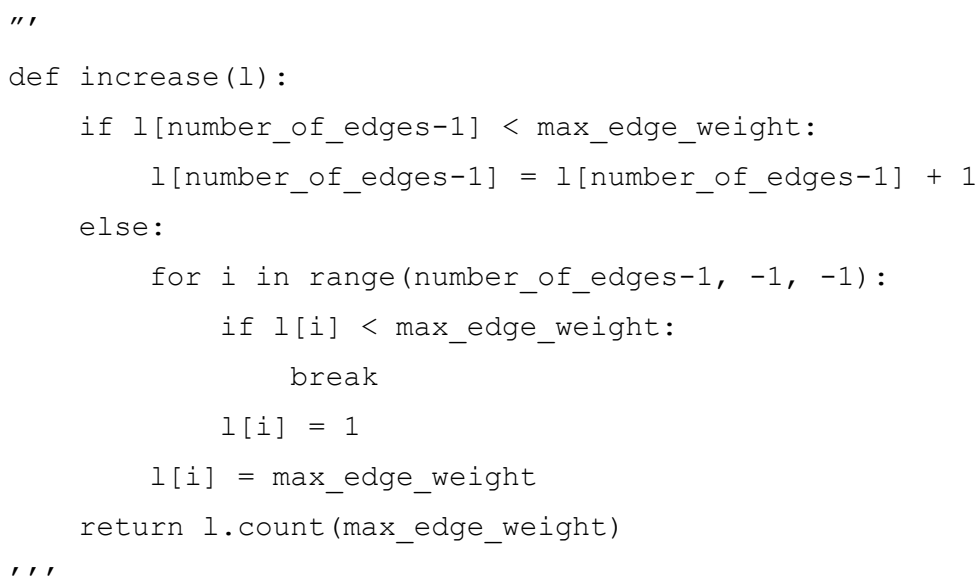

The function of is_valid tests the cases for DNA strands according to the Bead condition: the number of crossings on each face must be even.

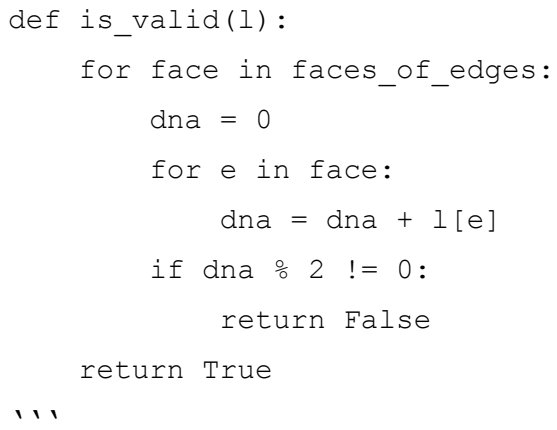

Produce the parameters of DNA bipyramid links satisfying the Bead condition.

$\mathrm{M}$ denotes the number of edges with even half-twists, while $\mathrm{N}$ denotes the number of edges with odd half-twists.

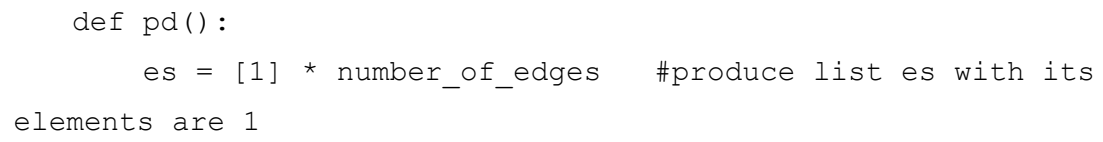




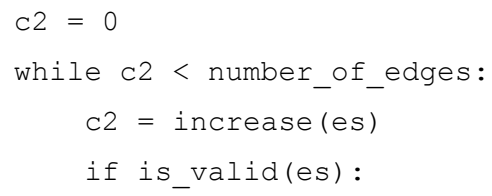

print (', '.join([" \{\}$=\{\}$ ".format ('abcdefghiMN'[k], x) for $k, x$ in enumerate (es+[c2, number_of_edges-c2])]))

$\operatorname{pd}()$

\section{Results and Discussion}

\subsection{Triangular bipyramid}

The program above obtains all possible cases for constructing the DNA triangular bipyramids. 16 projections of triangular bipyramids could be drawn according to these basic parameters. So, how many strands to construct the triangular bipyramids in each case would be clear. Adding attribute name: strands and organizing the data, Table 1 shows the parameters for constructing the DNA triangular bipyramids. The value of $a-i$ taking 1 means putting the odd half-twist on this edge, while 2 means the even half-twist. Odd denotes the number of edges with even half-twists, while even denotes the number of edges with odd half-twists.

Table 1. The parameters for constructing the DNA triangular bipyramids

\begin{tabular}{ccccccccccccc}
\hline No. & $a$ & $b$ & $c$ & $d$ & $e$ & $f$ & $g$ & $h$ & $i$ & odd & even & strand \\
\hline 1 & 1 & 2 & 1 & 1 & 2 & 2 & 1 & 1 & 1 & 6 & 3 & 2 \\
2 & 1 & 1 & 2 & 1 & 2 & 1 & 1 & 1 & 2 & 6 & 3 & 2 \\
3 & 1 & 2 & 1 & 2 & 1 & 1 & 1 & 2 & 1 & 6 & 3 & 2 \\
4 & 2 & 1 & 1 & 1 & 1 & 2 & 2 & 1 & 1 & 6 & 3 & 2 \\
5 & 1 & 1 & 2 & 1 & 2 & 1 & 2 & 2 & 1 & 5 & 4 & 2 \\
6 & 1 & 2 & 1 & 2 & 1 & 1 & 2 & 1 & 2 & 5 & 4 & 2 \\
7 & 1 & 1 & 2 & 2 & 1 & 2 & 2 & 1 & 1 & 5 & 4 & 2 \\
8 & 2 & 1 & 1 & 1 & 1 & 2 & 1 & 2 & 2 & 5 & 4 & 2 \\
9 & 2 & 2 & 2 & 1 & 1 & 1 & 1 & 2 & 1 & 5 & 4 & 2 \\
10 & 2 & 1 & 1 & 2 & 2 & 1 & 1 & 1 & 2 & 5 & 4 & 2 \\
11 & 1 & 1 & 2 & 2 & 1 & 2 & 1 & 2 & 2 & 4 & 5 & 4 \\
12 & 2 & 2 & 2 & 1 & 1 & 1 & 2 & 1 & 2 & 4 & 5 & 4 \\
13 & 2 & 1 & 1 & 2 & 2 & 1 & 2 & 2 & 1 & 4 & 5 & 4 \\
14 & 1 & 2 & 1 & 1 & 2 & 2 & 2 & 2 & 2 & 3 & 6 & 4 \\
15 & 2 & 2 & 2 & 2 & 2 & 2 & 1 & 1 & 1 & 3 & 6 & 4 \\
16 & 2 & 2 & 2 & 2 & 2 & 2 & 2 & 2 & 2 & 0 & 9 & 6 \\
\hline
\end{tabular}


There are still some equivalent ones, ambient isotopic in topology, in all 16 types derived from the program. To put it simply, isotopic links can be obtained from each other by applying symmetry operations. By means of mathematical or computer method, these isotopic links could be removed or filtered out ${ }^{[37,38,40]}$. After removing the isotopic links, 6 unique types of DNA triangular bipyramids remain, showed in Table 2, and 16 triangular bipyramids are classified into six groups: $\{\{1\},\{2,15\},\{3,5,10\},\{4,6,8,9,11,13\},\{7,12,14\},\{16\}\}^{[38]}$.

Table 2. The parameters for 6 unique DNA triangular bipyramids

\begin{tabular}{cccc}
\hline No. & odd & even & strand \\
\hline 1 & 6 & 3 & 2 \\
2 & 6 & 3 & 2 \\
3 & 5 & 4 & 2 \\
4 & 3 & 6 & 4 \\
5 & 4 & 5 & 4 \\
6 & 0 & 9 & 6
\end{tabular}

Intuitively, with the increase of edges with odd half-twists, the number of strands to construct triangular bipyramid decreases. Figure 5 shows the correlation between strands and edges with odd half-twists for the DNA triangular bipyramids (two data points overlap each other). Note that No. 1 and No. 2, in Table 2, are distinct types although they have the same parameters, and they cover up each other due to the same parameters in Table 2.

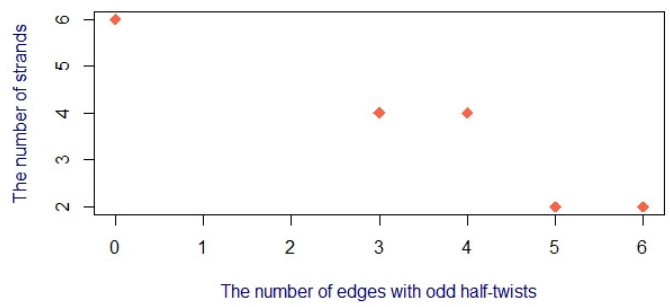

Figure 5. The correlation between the number of strands and the number of edges with odd half-twists for the DNA triangular bipyramids.

As we can see, the triangular bipyramids with minimum strands may be the one with maximum odd half-twists. Therefore, we select No.1 and No. 2 as the optimal types for the DNA triangular bipyramids. According to their parameters the program works out, we could draw up the two projections of the triangular bipyramids with minimum strands, shown in Figure 6. 


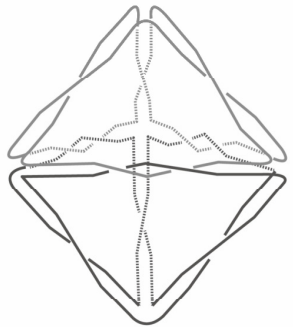

(a)

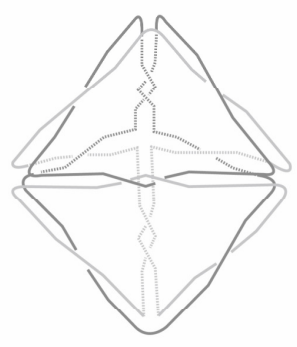

(b)

Figure 6. DNA triangular bipyramids with two strands.

\subsection{Pentangular bipyramid}

We also obtain the DNA pentangular bipyramid with minimum strands by using the same method. With the increase of structure complexity, the number of isotopic links of the pentangular bipyramid increases. Though, this does not conflict with the search for DNA pentangular bipyramid with minimum strands. After removing the isotopic links from 64 pentangular bipyramids, Table 3 shows the parameters for 12 unique DNA pentangular bipyramids.

Table 3. The parameters for 12 unique DNA pentangular bipyramids

\begin{tabular}{cccc}
\hline No & odd & even & strand \\
\hline 1 & 9 & 6 & 2 \\
2 & 10 & 5 & 2 \\
3 & 10 & 5 & 2 \\
4 & 10 & 5 & 4 \\
5 & 7 & 8 & 4 \\
6 & 8 & 7 & 4 \\
7 & 7 & 8 & 4 \\
8 & 6 & 9 & 6 \\
9 & 9 & 6 & 6 \\
10 & 5 & 10 & 6 \\
11 & 4 & 11 & 8 \\
12 & 0 & 15 & 10
\end{tabular}

Figure 7 shows the correlation between strands and edges with odd half-twists for the DNA pentangular bipyramids. Similarly, some distinct types having the same parameters may cover up each other, such as No.2 and No. 3. Generally, the number of strands is negatively correlated to the number of edges with odd half-twists, just as the fitted linear regression line in Figure 7 shows. 


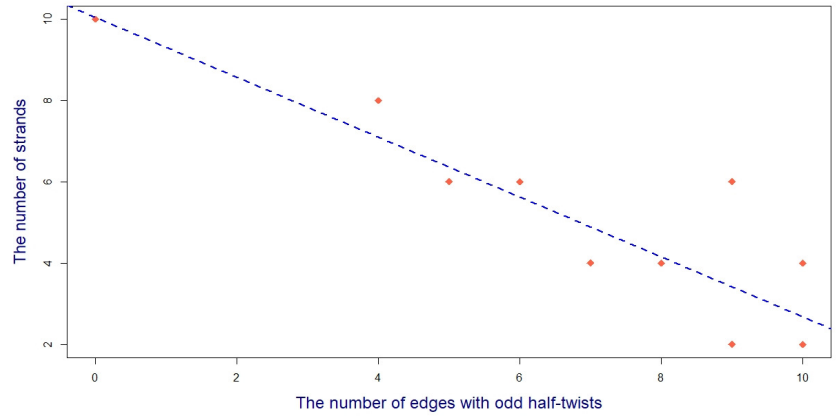

Figure 7. The correlation between the number of strands and the number of edges with odd half-twists for the DNA pentangular bipyramids.

As we see, the pentangular bipyramids with minimum strands may be the one with maximum odd half-twists. So, we select No. 2 and No. 3 as the optimal types for the DNA pentangular bipyramids. According to their parameters the program works out, we could draw up the projections of the pentangular bipyramids with two strands, shown in Figure 8.

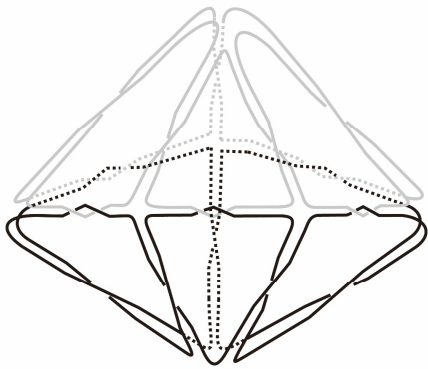

(a)

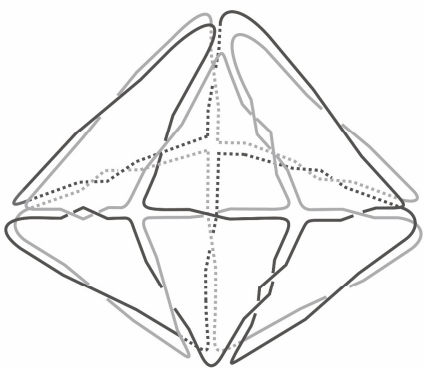

(b)

Figure 8. DNA pentangular bipyramids with two strands.

\section{Discussion}

Some general results about bipyramids can be obtained. It can be shown that no bipyramid can be constructed with a single DNA strand. A single DNA strand would correspond to an antiparallel strong trace. There is a theorem ${ }^{[41,42]}$ (Theorem 4.1 in [41] Theorem 2.4 in [42]) which says: 
A graph $G$ admits an antiparallel strong trace strong trace if and only if

$G$ has a spanning tree $T$ such that each connectedcomponent of $G-E(T)$ has an even number of edges.

Let $G$ be the skeleton of the triangular bipyramid (3-bipyramid). Since $|V(G)|=5$ and $|E(G)|=9$, the spanning tree $T$ has 4 edges, and the co-tree has 5 edges. It is clear that all connected components of the co-tree can not have an even number of edges. Therefore a single strand design does not exist. This can be generalised to an arbitrarily large bipyramid.

It is easy to show that arbitrarily large (2k+1)-bipyramid can be made of two DNA strands. Let $1,2,3, \ldots, 2 k+1$ be vertices of a cycle $C$. To obtain the bipyramid add vertices $a$ and $b$ and connected them to each vertex of the cycle $C$. Let the edges incident to $a$ and $b$ have odd halftwists and all the other edges have even half-twists. Then this results in a valid DNA design that is composed of two strands.

It is not hard to obtain numerical results for a few larger bipyramids (7-bipyramid, 9bipyramid) and plot the correlation between the number of strands and the number of odd halftwists. Perhaps this would lead to new interesting conjectures.

\section{Conclusions}

In this study, we theoretically propose an assembling strategy for DNA bipyramids with minimum strands based on a computer program. With two of three types of 3-, 4- and 5-degree vertexes, the triangular and pentangular bipyramids own more complex structure than Platonic solids. The result shows that the proposed assembling procedure could effectively search for DNA bipyramids with minimum strands. Meanwhile, the statistic analysis proves that the number of DNA strands is negatively correlated to the number of edges with odd half-twists. Although this strategy is theoretical, the techniques for designing and synthesizing cage-like structures are sophisticated. This theoretical study would enhance and help the design and synthesize of cages with complex pyramid-like structures.

Acknowledgements: This work is supported by the National Natural Science Foundation of China (No. 32060234), Gansu Provincial First-Class Discipline Program of Northwest Minzu University (No. 11080305), and Program for Innovative Research Team of State Ethnic Affairs Commission (SEAC) ([2018] 98). The authors are very thankful to the anonymous referees for their useful comments and suggestions, which helped us to improve this paper. 


\section{References}

[1] N. C. Seeman, DNA in a material world, Nature 421 (2003) 427-431.

[2] M. R. Jones, N. C. Seeman, C. A. Mirkin, Programmable materials and the nature of the DNA bond, Science 347 (2015) \#1260901.

[3] N. C. Seeman, Nanomaterials based on DNA, Annu. Rev. Biochem. 79 (2010) 65-87.

[4] R. P. Goodman, R. M. Berry, A. J. Turberfield, The single-step synthesis of a DNA tetrahedron, Chem. Commun. 10 (2004) 1372-1373.

[5] Z. Li, B. Wei, J. Nangreave, C. Lin, Y. Liu, Y. Mi, H. Yan, A replicable tetrahedral nanostructure self-assembled from a single DNA strand, J. Am. Chem. Soc. 131 (2009) 13093-13098.

[6] J. P. Sadowski, C. R. Calvert, D. Y. Zhang, N. A. Pierce, P. Yin, Developmental selfassembly of a DNA tetrahedron, ACS Nano 8 (2014) 3251-3259.

[7] W. M. Shih, J. D. Quispe, G. F. Joyce, A 1.7-kilobase single-stranded DNA that folds into a nanoscale octahedron, Nature 427 (2004) 618-621.

[8] Y. He, M. Su, P. Fang, C. Zhang, A. E. Ribbe, W. Jiang, C. Mao, On the chirality of selfassembled DNA octahedra, Angew. Chem. Int. 49 (2010) 748-751.

[9] C. L. P. Oliveira, S. Juul, H. L. Jøgensen, B. Knudsen, D. Tordrup, F. Oteri, M. Falconi, J. Koch, A. Desideri, J. S. Pedersen, F. F. Andersen, B. R. Knudsen, Structure of nanoscale truncated octahedral DNA cages: Variation of single stranded linker regions and influence on assembly yields, ACS Nano. 4 (2010) 1367-1376.

[10] F. Iacovelli, M. Falconi, B. R. Knudsen, A. Desideri, Comparative simulative analysis of single and double stranded truncated octahedral DNA nanocages, RSC Adv. 6 (2016) 35160-35166.

[11] C. M. Erben, R. P. Goodman, A. J. Turberfield, A self-assembled DNA bipyramid, J. Am. Chem. Soc. 129 (2007) 6992-6993.

[12] X. He, L. Dong, W. Wang, N. Lin, Y. Mi, Folding single-stranded DNA to form the smallest 3D DNA triangular prism, Chem. Commun. 49 (2013) 2906-2908.

[13] Z. Nie, X. Li, Y. Li, C. Tian, P. Wang, C. Mao, Self-assembly of DNA nanoprisms with only two component strands, Chem. Commun. 49 (2013) 2807-2809.

[14] N. Xie, S. Liu, X. Yang, X. He, J. Huang, K. Wang, DNA tetrahedron nanostructures for biological applications: biosensors and drug delivery, Analyst 142 (2017) 3322-3332.

[15] Y. Hu, Z. Chen, H. Zhang, M. Li, Z. Hou, X. Luo, X. Xue, Development of DNA tetrahedron-based drug delivery system, Drug Delivery 24 (2017) 1295-1301. 
[16] Q. Hu, H. Li, L. Wang, H. Gu, C. Fan, DNA nanotechnology-enabled drug delivery systems, Chem. Rev. 119 (2019) 6459-6506.

[17] A. Tay, N. Melosh, Nanostructured materials for intracellular cargo delivery, Acc. Chem. Res. 52 (2019) 2462-2471.

[18] J. Liu, L. Song, S. Liu, Q. Jiang, Q. Liu, N. Li, Z. G. Wang, B. Ding, A DNA-based nanocarrier for efficient gene delivery and combined cancer therapy, Nano Lett. 18 (2018) 3328-3334.

[19] H. Liu, Y. Chen, Y. He, A. E. Ribbe, C. Mao, Approaching the limit: can one DNA oligonucleotide assemble into large nanostructures? Angew. Chem. Int. Ed. 45 (2006) 1942-1945.

[20] C. Tian, C. Zhang, X. Li, C. Hao, S. Ye, C. Mao, Approaching the limit: can one DNA strand assemble into defined nanostructures? Langmuir 30 (2014) 5859-5862.

[21] J. Duan, L. Cui, Y. Wang, H. Zheng, An approach to generate DNA polyhedral links of one/two strands, J. Mol. Graph. Model. 97 (2020) 107565.

[22] J. Duan, L. Cui, Y. Wang, Rational design of DNA platonic polyhedra with the minimal components number from topological perspective, Biochem. Bioph. Res. Commun. $\mathbf{5 2 3}$ (2020) 627-631.

[23] J. Li, D. Jiang, B. Bao, Y. He, L. Liu, X. Wang, Radiolabeling of DNA bipyramid and preliminary biological evaluation in mice, Bioconjugate Chem. 27 (2016) 905-910.

[24] W. Qiu, X. Zhai, Y. Qiu, Architecture of Platonic and Archimedean polyhedral links, Sci. China Ser. B Chem. 51 (2008) 13-18.

[25] G. Hu, X. Zhai, D. Lu, W. Qiu, The architecture of Platonic polyhedral links, J. Math. Chem. 46 (2009) 592-603.

[26] W. Qiu, Z. Wang, G. Hu, The Chemistry and Mathematics of DNA Polyhedra, Nova Sci., New York, 2010.

[27] G. Hu, W. Qiu, A. Ceulemans, A new Euler's formula for DNA polyhedra, PLoS One 6 (2011) \#e26308.

[28] T. Deng, M. Yu, G. Hu, W. Qiu, The architecture and growth of extended Platonic polyhedra, MATCH Commun. Math. Comput. Chem. 67 (2012) 713-730.

[29] T. Deng, W. Qiu, The architecture of extended Platonic polyhedral links, MATCH Commun. Math. Comput. Chem.70 (2013) 347-364.

[30] J. Duan, G. Hu, and W. Qiu, Topological aspect of DNA cages: Genus, MATCH Commun. Math. Comput. Chem. 72 (2014) 475-488. 
[31] J. Duan, W. Li, X. Li, G. Hu, W. Qiu, Molecular design of DNA polyhedra based on genus, J. Math. Chem. 52 (2014) 2380-2394.

[32] T. Deng, J. Duan, W. Li, W. Qiu, A new Euler formula and its characterization of DNA polyhedra, MATCH Commun. Math. Comput. Chem. 75 (2016) 387-402.

[33] L. Guo, H. Bai, J. Hao, S. Liu, A topological approach to assembling strands-based DNA triangular prisms, MATCH Commun. Math. Comput. Chem. 82 (2019) 219-253.

[34] T. Sawada, A. Saito, K. Tamiya, K. Shimokawa, Y. Hisada and M. Fujita, Metalepeptide rings form highly entangled topologically inequivalent frameworks with the same ringand crossing-numbers, Nat. Commun. 10 (2019) 921.

[35] N. Jonoska, R. Twarock, Blueprints for dodecahedral DNA cages, J. Phys. Math. Theor. 41 (2008) \#304043.

[36] N. E. Grayson, A. Taormina, R. Twarock, DNA duplex cage structures with icosahedral symmetry, Theor. Comput. Sci. 410 (2009) 1440-1447.

[37] T. Deng, Configuration of DNA polyhedra of truncated tetrahedron, cuboctahedron, truncated octahedron, J. Theor. Biol. 472 (2019) 4-10.

[38] T. Deng, Construction and analysis of double helix for triangular bipyramid and pentangular bipyramid, Comput. Math. Method. M. 2020 (2020) \#5609593.

[39] W. Qiu, X. Zhai, Molecular design of Goldberg polyhedral links, J. Mol. Struct. (Theochem) 756 (2005) 163-166.

[40] S. Liu, L. Guo, H. Bai, J. Hao, A topological approach to assembling strands-based DNA tetrahedra, MATCH Commun. Math. Comput. Chem. 81 (2019) 209-244.

[41] G. Fijavž, T. Pisanski, J. Rus, Strong traces model of self-assembly polypeptide structure, MATCH Commun. Math. Comput. Chem. 71 (2014) 199-212.

[42] J. Rus, Antiparallel d-stable traces and a stronger version of ore problem, J. Math. Biol. 75 (2017) 109-127. 